\title{
Proceedings of the 34th Annual Upper Midwest Neuro-Ophthalmology Group Meeting, July 26, 2013, Chicago, Illinois, USA
}

\author{
Evan B. Price ${ }^{1}$, Heather E. Moss ${ }^{1}$, and Ivy Dreizin ${ }^{2}$ \\ ${ }^{1}$ Division of Neuro-Ophthalmology, Department of Ophthalmology and Visual Sciences, University of Illinois, \\ Chicago, Illinois, USA and ${ }^{2}$ Department of Neurology, University of Wisconsin Medical Foundation, Madison, \\ Wisconsin, USA
}

The 34th annual meeting of the Upper Midwest Neuro-Ophthalmology Group (UMNOG) was held on July 26, 2013, at Northwestern University, Chicago, Illinois. Thirty participants, including four fellows and one resident physician, benefited from the collegiality and spirited discussion that have characterised this forum since its inception. The organisation and direction of the event were performed by Dr. Ivy Dreizin, University of Wisconsin, Madison, Wisconsin, who also expertly kept the pace of the proceedings moving forward.

Dr. Walter Jay, Loyola University, Maywood, Illinois, began the day with a case of Horner's syndrome in a patient with neuromyelitis optica (NMO). Her condition was confirmed by a positive apraclonidine test, the presence of anti-aquaporin-4 antibodies, and a criteria-meeting, contrast-enhancing lesion of the cervical spinal cord. Discussion focused on the rarity of such a presentation and the question was raised why Horner's syndrome is not noted as frequently with cervical cord lesions as one would expect, given the typical course of the sympathetic pathway from the hypothalamus to the pupil.

The discussion of NMO continued with a presentation by Dr. Bonnie Keung, University of Illinois at Peoria, Peoria, Illinois, that stimulated a lively discussion on the nature of NMO spectrum disorder. The patient, who was just 12 years old at presentation, had a positive anti-aquaporin- 4 antibody test and prominent enhancing lesions of the diencephalon and brainstem, but no optic nerve or spinal cord involvement. Biopsy of the lesion done rapidly after initial onset of symptoms did not show characteristic demyelination, a fact that brought about discussion of the nature of NMO spectrum disorder itself.

Dr. Robert Tomsak, Kresge Eye Institute, Detroit, Michigan, presented a case of a 66-year-old patient with bilateral, sequential non-arteritic ischaemic optic neuropathy (NAION). The ongoing search for effective treatment of NAION was the central focus of the group's discussion, and the possible utility of erythropoietin or memantine sparked discussion that continued over e-mail since the meeting.

Dr. Rashmi Varma, neuro-ophthalmology fellow, University of Michigan, Ann Arbor, Michigan, presented the case of a 33-year-old man status post motor vehicle accident with facial fracture and delayed onset of periocular pain, bruising, chemosis, subconjunctival haemorrhage, and manifest esotropia. Although carotid-cavernous fistula was initially suspected, an angiogram revealed no such pathology. Due to increased watery discharge from the eye upon leaning forward, a diagnosis of cranio-orbital fistula with occulorhoea was considered. This was confirmed via subconjunctival fluid aspiration, which revealed the presence of beta-2 transferrin. The importance of 
considering such a rare condition was discussed, as well as its concordant risk of meningitis.

Dr. Jacqueline Leavitt, Mayo Clinic, Rochester, Minnesota, then took to the floor to discuss the Mayo Clinic experience regarding permitting non-ophthalmologists to order visual field testing and interpretation without eye examination. Preliminary results indicate that requesting providers are using the "visual field with interpretation only" order properly for the vast majority of field requests.

Dr. Crandall Peeler, ophthalmology resident, University of Michigan, Ann Arbor, Michigan, presented a series of cases highlighting the utility of diffusion-weighted imaging (DWI) in the care of patients with various non-ischaemic pathologies of the brain and orbit. In each case, careful analysis of the DWI examination altered the patient's management. In two patients, occult subretinal abscesses were identified and subsequently treated. In another, a cavernous sinus thrombosis was discovered, ostensibly obviating the need for venography.

Dr. Timothy Kietzman, neuro-ophthalmology fellow, Northwestern University, Chicago, Illinois, presented a series of patients with Susac's syndrome, putting emphasis on recognising the stigmata of this rare disease. The ensuing discussion focused on not only the futility of our current management strategies, but also the story of Dr. Susac himself and his relationship with the condition that bears his name.

Dr. Steven Stasheff, University of Iowa, Iowa City, Iowa, reported on his fascinating research in the rd12 mouse, an RPE65-deficient model of Leber's congenital amaurosis (LCA). Using in vitro multielectrode recording of the mouse retina, Dr. Stasheff's team is bringing new clarity to our understanding of how gene therapy for LCA alters the neural code transmitted by the retinal ganglion cells (RGCs). As compared with negative controls, recovery of light-evoked responses was demonstrated in treated mice only. However, spontaneous RGC hyperactivity was also present at levels similar to those seen in untreated $r d 12$ mice. Dr. Stasheff shared that it is possible that gene therapy earlier in the life of the mouse may reduce this background hyperactivity while retaining robust light-evoked responses.

To start the afternoon, Dr. Dane Brecker, neuroophthalmology fellow, University of Michigan, Ann Arbor, Michigan, presented the case of a patient with persistent homonymous hemianopsia after focal status epilepticus. He had a magnetic resonance imaging (MRI) of the brain that was read as normal, but upon close inspection displayed a possible structural lesion. The illuminating group discussion focused on MRI findings in seizure.
Dr. Jorge Kattah, University of Illinois at Peoria, Peoria, Illinois, described two Wernicke's encephalopathy patients with upbeat nystagmus that spontaneously converted to downbeat nystagmus. This included an excellent discussion of possible causative mechanisms.

Dr. Nicholas Volpe, Northwestern University, Chicago, Illinois, discussed his research on surgical correction for hypotropia in thyroid eye disease. The authors used an adjustable suture technique, aiming for undercorrection in primary gaze without hyperdeviation in downgaze, in an effort to preempt drift toward overcorrection of recessed inferior recti. They were able to effectively manage hypotropia with one surgery in the vast majority of patients.

An intriguing case was then presented by Dr. Jonathan Trobe, University of Michigan, Ann Arbor, Michigan. The patient was a middle-aged gentleman who presented with headache and was found to have papilloedema, normal imaging of the brain, and elevated opening pressure on lumbar puncture. He was diagnosed with idiopathic intracranial hypertension, but was unable to tolerate treatment with acetazolamide because of side effects. On three separate occasions over the following year, he presented with cerebrospinal fluid (CSF) rhinorrhoea. At each subsequent encounter, a discontinuity was found in the cribiform bone, then the temporal bone, and finally the sphenoid bone. Each of these defects was repaired after diagnosis. A ventriculoperitoneal shunt was placed after this final episode for presumed persistently elevated intracranial pressure (ICP), and he has been well since. This case was illustrative of the noted association between elevated ICP and CSF leak, and gave credence to the argument for ophthalmoscopic evaluation of CSF leak patients for possible papilloedema.

Dr. Tomzak returned to the podium to present a patient with an optic neuropathy of one eye, possibly posterior ischaemic optic neuropathy (PION) from optic disc drusen (ODD). The discussion focused on the possibility of such a causative mechanism for PION, highlighting the fact that NAION in ODD has been described, but that a PION from ODD would be a novel clinical entity.

The final presentation of the day was made by Dr. Roy Meckler, Louisville, Kentucky, who began with some remarks on the history of UMNOG. His case presentation closed the day the way it beganwith a discussion of NMO and NMO spectrum disorder. The pervasiveness of these devastating conditions in the day's discourse reflects the current interests of the field at large and the active research investigating their nature and treatment.

UMNOG 2013 brought together interested physicians and researchers from across the midwestern United States in the common bond of a commitment 
to both scientific excellence and respectful, genial discourse. Thanks go to Dr. Nicholas Volpe for hosting the event and to all attendees for making the meeting such a valuable way to spend the last Friday in July each year. All who are interested in attending UMNOG next year or would like to be included on the UMNOG mailing list should e-mail Dr. Ivy Dreizin at ivy.dreizin@uwmf.wisc.edu.

Declaration of interest: The authors report no conflicts of interest. The authors alone are responsible for the content and writing of the paper. 